\title{
RDUS
}

Revue de DROIT

UNIVERSITÉ DE SHERBROOKE

Titre : $\quad$ LE SUIVI DE L'ÉTHIQUE DES PROJETS DE RECHERCHE EN GÉNÉTIQUE

Auteur(s): $\quad$ Mylène DESCHÊNES

Revue: $\quad$ RDUS, 2004-2005, volume 35, numéro 1

Pages: $\quad$ 187-229

ISSN : $\quad 0317-9656$

Éditeur : $\quad$ Université de Sherbrooke. Faculté de droit.

URI : $\quad$ http://hdl.handle.net/11143/11932

DOI : https://doi.org/10.17118/11143/11932 
Page vide laissée intentionnellement. 
ARTICLE

\section{LE SUIVI DE L'ÉTHIQUE DES PROJETS DE RECHERCHE EN GÉNÉTIQUE}

\section{par Mylène DESCHÊNES*}

La nécessité d'effectuer un suivi de l'éthique des projets de recherche s'impose comme un élément-clé dans la protection des participants en recherche. Cependant, les responsabilités précises de chacun des intervenants de même que les moyens qu'il convient de mettre en ouvre pour s'acquitter de cette tâche ne sont pas clairement énoncés. De plus, le suivi de la recherche en génétique pose des problématiques particulières qui ont reçu peu d'échos dans la littérature jusqu'à présent.

Dans ce texte, l'auteure aborde le sujet du suivi de l'éthique des projets de recherche en débutant par un examen de la terminologie qui laisse entrevoir une certaine confusion au sujet des concepts en présence. Ensuite, l'auteure se penche sur la nécessité pour les comités d'éthique de la recherche d'effectuer un suivi de l'éthique des projets qu'ils approuvent et fait la recension du fondement normatif de l'obligation de suivi éthique au niveau international et national.

Dans la seconde partie du texte, l'auteure examine plus particulièrement les défis soulevés par le suivi éthique de projets de recherche en génétique. L'auteure note qu'il est nécessaire d'ajuster la mire et de se concentrer non seulement sur l'intégrité physique du participant mais surtout sur la vie privée et la dignité de ce dernier. L'auteure décrit aussi les difficultés particulières auxquelles sont confrontés ces comités lorsqu'ils tentent d'effectuer un suivi de l'éthique de projets de recherche en génétique faisant appel à des banques d'ADN contenant des échantillons provenant de multiples institutions ou conservés dans des institutions étrangères. Cette analyse met en lumière les limites du CÉR à pouvoir assumer cette responsabilité à l'égard de certains projets. Enfin, l'auteure fait une liste de recommandations pratiques qui pourraient aider les CÉR à y voir plus clair dans leurs activités de suivi pour des projets de recherche en génétique.

*. L'auteure est avocate et diplômée en droit de l'Université McGill et a complété une Maîtrise en droit des biotechnologies à l'Université de Montréal. Elle est agent de recherche et directrice de projets au Centre de recherche en droit public de l'Université de Montréal. Elle siège à différents comités d'éthique de la recherche dont celui de l'Institut de cardiologie de Montréal. Cet article est l'un des fruits d'un projet subventionné par les Instituts de recherche en santé du Canada (anciennement le CRM [\# FRN-36059]). L'équipe de recherche est composée de Bartha Maria Knoppers, Kathleen Cranley Glass et Hubert Doucet. L'article fut préparé grâce à la collaboration de Mireille Brisson, pour la recherche sur les aspects internationaux, ainsi que l'assistance de Claudine Fecteau, Alana Greenberg, Gabrielle Grégoire et Simone Truong. De plus, l'auteure tient à remercier le Projet génétique et société, le Multidisciplinary Human Gene Technology, Research and Therapy Project Scholarship de même que les membres du Research Center for Genetic Medicine, Children's National Medical Center, Washington DC. 
The ongoing surveillance of research projects is a key element in protecting those who participate in research. The specific responsibilities of each participant, as well as an indication of the means by which adequate surveillance may be ensured are not however clearly enunciated. Moreover, genetic research raises specific problems which have not been sufficiently dealt with in related literature.

In this article, the writer deals with the continuing review of the ethical aspects of research projects,. She begins with an examination of the pertinent terminology and points out that there exits some confusion surrounding the concepts involved. The writer then alludes to the necessity that research ethics boards (REBs) ensure the ongoing ethical validity of projects which they have approved. She describes the national and international normative foundations of the duty to provide monitoring of the ethical aspects of research in progress.

The second part of this paper deals more particularly with the challenges arising from the need to monitor genetic research. It is suggested that the goals should be modified to focus not only on the protection of the physical integrity of the participants, but also to emphasize the need to protect their right of privacy and their dignity. The writer describes certain difficulties faced by research ethics boards when they review genetic research projects involving DNA biobanks which collect samples from multiple sites or which are set up in institutions other than those with which the research ethics boards in question are affiliated. This analysis reveals the limitations of research ethics boards to properly assume their responsibilities with regard to certain projects. The article concludes with practical recommendations which could be of help to research ethics boards in their monitoring of genetic research projects. 


\section{SOMMAIRE}

Introduction

Partie I : $\quad$ Le suivi de l'éthique des projets de recherche . . . . . 193

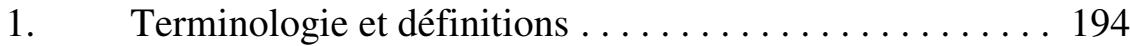

2. Nécessité d'assurer un suivi de l'éthique et bilan . . . . . 198

3. Fondements normatifs de l'obligation de suivi ....... 201

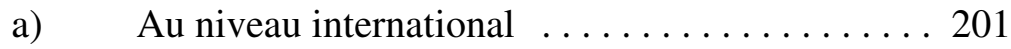

b) Au niveau national et provincial . . . . . 202

Conclusion de la Partie $I \ldots \ldots \ldots \ldots \ldots \ldots$

Partie II : Le suivi de l'éthique dans le contexte particulier de la recherche en génétique humaine . . . . . . . . . 209

1. La pertinence d'un suivi et d'une surveillance éthique dans la recherche en génétique humaine ......... 211

2. Fixer le point de mire du suivi de l' éthique des projets de recherche en génétique . . . . . . . . . . . . 212

3. Un cas particulièrement difficile : La recherche «multicentre» en génétique ou effectuée à partir d'une banque externe ..................... 215

a) Un problème de contrôle et d'information ..... 217

b) Un problème d'encadrement normatif . . . . . . 218

c) Problème de sanction . . . . . . . . . . 220

4. Recommandations pratiques pour le suivi de l'éthique des projets de recherche institutionnelle en génétique humaine . . . . . . . . . . . . . . . . . 221

a) Prélude à la surveillance : l'identification des banques d'ADN existantes et leurs politiques respectives ................... 221

b) Le respect des engagements pris à l'égard de la conservation des échantillons . . . . . . . . . . . 223

c) Le respect des engagements pris à l'égard du déroulement de la recherche et de l'utilisation des échantillons . . . . . . . . . . . . . . . . . . 225 
d) Le respect des engagements pris à l'égard des résultats de recherche .............. 226

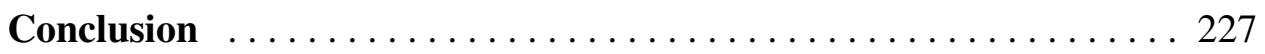



unquestioning trust, nor can it be assumed that scientists are different from other human beings and totally incapable of error, deceit, misrepresentation, or bias. The scientific community must be vigilant for this, since nothing less than the viability of the biomedical science enterprise is at stake.» ${ }^{1}$

\section{Introduction}

La surveillance des projets de recherche préoccupe de plus en plus tous les intervenants du milieu de la recherche. Alors que l'approbation des projets de recherche par un comité d'éthique de la recherche [Ci-après le CÉR] permet d'examiner, avant le début du projet, si la sécurité des participants et le respect des principes éthiques et légaux apparaissent adéquats, on ne saurait s'en satisfaire quant à l'ensemble du déroulement d'un projet. Pour veiller adéquatement au déroulement éthique de projets de recherche, il faut faire un pas de plus et assurer un suivi de l'ensemble de la mise en œuvre d'un projet de recherche.

La nécessité d'effectuer une surveillance des projets de recherche s'impose comme un élément-clé dans la protection des participants en recherche. Cependant, les responsabilités précises de chacun des intervenants de même que les moyens qu'il convient de mettre en œuvre pour s'acquitter de cette tâche ne sont pas clairement énoncés. Dans le domaine de la recherche pharmaceutique traditionnelle, on a observé l'inclusion progressive de diverses pratiques relatives à la surveillance des projets de recherche. Certaines sont le fruit d'initiatives locales ${ }^{2}$ et $d$ 'autres mesures ont acquis un caractère obligatoire

1. R.P. Shwarz, «Maintaining Integrity and Credibility in Industry-Sponsored Clinical Research» (1991) 12 Controlled Clin. Trials 753 à la p. 759.

2. M. Deschênes, P. Deschamps et R. Martineau, «La surveillance éthique continue des projets de recherche en génétique; de la théorie à la pratique» dans C. Hervé, B. M. Knoppers et $\mathrm{P}$. Molinari, dir., Les pratiques de recherche biomédicale visitées par la bioéthique, Paris, Dalloz, 2003, 27. 
grâce à des documents normatifs tels que les Bonnes pratiques cliniques $^{3}$. Malgré qu'on en reconnaisse toute l'importance, force est de constater que les activités de surveillance sont insuffisantes. C'est d'ailleurs le constat que le Vérificateur général du Québec dressait dans un rapport réalisé en $2001^{4}$. En somme, la pleine concrétisation du suivi de l'éthique des projets de recherche dans le domaine pharmaceutique demeure encore à réaliser.

Qu'en est-il de la génétique? En fait, personne ne semble lui avoir accordé une attention particulière. Nos recherches, à l'échelle nationale et internationale, ne nous ont pas permis de trouver des lignes directrices précises sur cette question. Dans un contexte où on a déjà du mal à concrétiser pleinement la notion de suivi dans le domaine de la recherche pharmaceutique, il n'est pas étonnant qu'on ne se soit pas encore penché sur le cas spécifique de la recherche en génétique.

Pourtant, les enjeux sont importants. Il a été maintes fois réitéré que la recherche en génétique comporte des particularités qui ne correspondent pas au modèle traditionnel de recherche. C'est ainsi, par exemple, que le modèle des formulaires de consentement traditionnellement employé en recherche pharmaceutique a été revu et adapté aux réalités de la génétique ${ }^{5}$. De plus, la recherche en génétique prend une ampleur sans précédent. On voit naître des projets de recherche forts ambitieux ayant recours à des vastes banques d'ADN, tant publiques que privées, qui sont souvent le fait de plus d'une institution, et qui sont couplées à diverses données personnelles. Compte tenu de cette nouvelle réalité, il est opportun de se questionner sur le processus de suivi approprié.

Notre analyse est divisée en deux parties. Dans la première partie, nous discuterons de la surveillance en général. Dans la seconde partie, nous nous

3. Santé Canada, Directive tri-partite normalisée des ICH. Les bonnes pratiques cliniques : directives consolidées, Ottawa, Travaux publics et services gouvernementaux Canada, 1997 [ci-après les Bonnes pratiques cliniques].

4. Québec, Vérificateur général, Rapport à l'Assemblée nationale pour l'année 2000-2001, t. 1, Québec, Publications du Québec, 2001 à la p. 82 [ci-après Vérificateur général, Rapport].

5. M. Meschênes et al., «Human Genetic Research: DNA Banking and Consent: a Question of "form"?» (2001) 59:4 Clinical Genetics 221. 
pencherons plus particulièrement sur le suivi de l'éthique des projets de recherche en génétique. Nous identifierons quelques défis de taille auxquels les CÉR doivent faire face. Nous formulerons des recommandations pratiques applicables aux projets institutionnels de recherche en génétique.

Nous tenons à préciser que le présent document a pour but d'étudier la surveillance qui doit s'exercer sur des projets de recherche en génomique. Il ne s'agit donc pas de bonifier l'ensemble de la doctrine sur les activités de surveillance qui existent dans le domaine pharmaceutique mais plutôt d'identifier les ajustements requis afin d'assurer une surveillance adéquate dans le domaine particulier de la génétique. Il faut noter que notre propos ne portera pas sur le suivi de projets de recherche en thérapie génique et xénotransplantation, compte tenu que ces projets soulèvent des enjeux particuliers $^{6}$. De même, nous n'examinerons pas les aspects particuliers concernant les mineurs et les inaptes (comme par exemple, ceux liés au suivi de la divulgation de résultats personnels à l'âge adulte). Notre attention portera plutôt sur les projets de recherche en génétique, qu'ils soient individuels ou familiaux, en pharmacogénomique, ainsi que sur les banques D'ADN.

\section{Partie I : $\quad$ Le suivi de l'éthique des projets de recherche}

Avant d'entreprendre l'examen du suivi de l'éthique des projets de recherche en génétique, voici un tour d'horizon de la situation en ce qui concerne le suivi général des projets de recherche. Premièrement, nous étudierons la terminologie employée dans ce domaine. Deuxièmement, nous discuterons de la nécessité de faire un tel suivi. Troisièmement, nous parlerons du fondement normatif de l'obligation de surveillance éthique continue.

6. Une combinaison complexe de problèmes liés à la surveillance à long terme et à la santé publique. 


\section{Terminologie et définitions}

À la lecture de la doctrine portant sur la surveillance des projets de recherche, on constate rapidement qu'il règne une certaine confusion dans le vocabulaire, qui est d'ailleurs aggravée lorsqu'on traduit de l'anglais au français. La doctrine parle tour à tour d'évaluation continue, de surveillance, de vérification, de suivi, de «monitoring», d'examen continu, etc... Or, peu de documents font une classification claire de ces différentes activités. Nous croyons qu'une partie de la confusion liée à la surveillance éthique continue est directement attribuable au fait que certains emploient le même mot pour désigner des concepts différents. Un autre problème réside dans le fait que, audelà de la sémantique, ces diverses activités sont mal balisées, laissant bien des zones obscures.

Pour minimiser la confusion dans le propos du présent texte, nous débuterons donc en faisant un tour d'horizon du vocabulaire employé. Quelques définitions sont proposées par les Bonnes pratiques cliniques. De plus, le vocabulaire employé a fait l'objet d'une analyse plus approfondie par le Groupe de travail sur l'examen continu des Instituts de recherche en santé du Canada [ci-après, Groupe de travail sur l'examen continu]. Ce dernier propose de subdiviser les activités de suivi en trois groupes distincts.

L'évaluation continue décrit l'activité qui incombe au comité d'éthique de veiller à ce que sa décision demeure adéquate et pertinente en regard de l'évolution potentielle d'un protocole, de sa mise en œuvre et des connaissances scientifiques au cours du déroulement d'un projet. Ce terme n'est pas défini dans les Bonnes pratiques cliniques. Néanmoins, le Groupe de travail sur l'examen continu en fait la définition suivante :

L'Examen (sic) continu du protocole par le CÉR désigne ici les capacités qui doivent exister pour tous les protocoles de recherche avec des sujets humains pour veiller à ce que l'approbation de la recherche par le CÉR continue de reposer sur la meilleure information à jour.

L'examen continue par le CÉR des facteurs scientifiques et éthiques sur lesquels reposait l'approbation du CÉR est essentiel pour toute la 
recherche avec des sujets humains. La justification continue du recours à des humains dans le projet de recherche et l'information sur laquelle s'appuie leur consentement à y participer sont les principaux objets de l'examen continu par le CÉR.?

Cette évaluation se fonde principalement sur les données offertes par le chercheur. Il va sans dire que l'équipe de recherche assume donc la responsabilité de fournir régulièrement des informations à jour et validées au CÉR afin de lui permettre de prendre des décisions éclairées et d'actualiser les décisions prises au besoin. On pourrait aussi employer les termes «examen continu du protocole de recherche», «suivi», «suivi éthique» ou encore «suivi de l'éthique» comme synonymes.

La surveillance (en anglais le «monitoring») est définie dans les Bonnes pratiques cliniques de la façon suivante:

Activité qui consiste à surveiller le déroulement d'un essai clinique et à veiller à ce que cet essai soit réalisé conformément au protocole, aux modes opératoires normalisés (MON), aux bonnes pratiques cliniques (BPC) et aux exigences réglementaires applicables, et que les données s'y rattachant soient consignées et présentées conformément à ces mêmes directives. ${ }^{8}$ (nos italiques)

La différence entre l'évaluation continue et la surveillance est subtile. Le Groupe de travail suggère que la surveillance diffère de l'évaluation continue par le fait qu'elle ne s'exerce que sur certains protocoles en fonction des besoins, alors que l'évaluation continue est une préoccupation constante dans l'approbation et le déroulement de tous les protocoles de recherche ${ }^{9}$. Le mot «surveillance» est par ailleurs chargé d'un regard inquisiteur qui peut mettre certaines personnes sur la défensive. Il faut aussi noter que le terme «surveillance continue» est parfois employé dans un sens qui se rapproche plus de la notion de «l'évaluation continue» préalablement décrite.

7. GROUPE DE TRAVAIL SUR L'EXAMEN CONTINU DES INSTITUTS DE RECHERCHE EN SANTÉ DU CANADA, «Projet de rapport» (2000) à la p. 5 [ci-après Groupe de travail].

8. $\quad$ Bonnes pratiques cliniques, supra note 3, sec. 1.38 .

9. $\quad$ Groupe de travail, supra note 7 à la p. 5. 
Le fait que la responsabilité inhérente à chacun de ces deux concepts relève de personnes différentes constitue une autre distinction importante. Alors que l'évaluation éthique est clairement l'apanage du CÉR, il n'en va pas de même de la surveillance continue. Tous ne s'entendent pas sur les personnes qui doivent s'occuper de la surveillance ${ }^{10}$. Selon le Groupe de travail sur l'examen continu, tous les acteurs impliqués dans la recherche peuvent prendre part à une activité de surveillance ${ }^{11}$. Celle-ci peut prendre plusieurs formes dont, par exemple, le contrôle exercé par le comité indépendant de contrôle des données (Data Safety Monitoring Board aussi appelé par son acronyme anglais : DSMB). Cependant, les auteurs des Bonnes pratiques cliniques ne semblent pas être du même avis. En effet, si on se fie à leurs propos, la surveillance semble être une activité sous la responsabilité du promoteur.

Les surveillants doivent être nommés par le promoteur. ${ }^{12}[\ldots]$

Le promoteur doit veiller à ce que les essais soient surveillés adéquatement. ${ }^{13}[\ldots]$

On pourrait penser qu'il y a là confusion avec l'audit. Toutefois, l'audit fait l'objet d'une définition distincte dans les Bonnes pratiques cliniques. De plus, à notre surprise, l'Énoncé du politique des trois Conseils indique que sauf exception, la surveillance continue de l'éthique des projets de recherche ne devrait pas être effectuée par les CÉR ${ }^{14}$.

Par ailleurs, une vérification (audit) est définie dans les Bonnes pratiques cliniques comme :

10. Pour une recension des divers points de vue à ce sujet et une analyse sur la question, voir S. Audy, La surveillance continue de l'éthique des projets de recherche : problématique et repères éthiques, Comité de liaison en éthique de la recherche de l'Université de Montréal, (1999). L'auteure se montre sceptique quant au rôle que peut jouer le CÉR dans la surveillance des projets de recherche.

11. Groupe de travail, supra note 7 à la p. 6.

12. Bonnes pratiques cliniques, supra note 3, art. 5.18.2.

13. Ibid., art. 5.18.3.

14. Conseil de recherches médicales du Canada, Conseil de recherches en sciences naturelles et en génie du Canada, Conseil de recherches en sciences humaines du Canada (IRSC, CRSN et CRSH), Énoncé de politique des trois Conseils : Éthique de la recherche avec des êtres humains, Ottawa, 1998, règle 1.13. [Ci-après Énoncé de politique] 
Examen systématique et indépendant des activités et documents liés aux essais en vue de déterminer, d'une part, si ces activités ont été menées conformément au protocole, aux modes opératoires normalisés $(\mathrm{MON})$ du promoteur, aux bonnes pratiques cliniques (BPC) et aux exigences réglementaires applicables et, d'autres part, si les données ont été enregistrées, analysées et présentées conformément à ces mêmes directives. ${ }^{15}$ (nos italiques)

Les Bonnes pratiques cliniques exigent que le promoteur nomme des individus indépendants de la recherche afin de mener l'audit ${ }^{16}$. Le Groupe de travail sur l'examen continu indique que les vérifications ont pour but de s'assurer de la qualité de la recherche, de l'éducation des équipes de recherche ainsi que de la protection des sujets de recherche ${ }^{17}$. Contrairement aux Bonnes pratiques cliniques, ce comité est plutôt d'avis que la responsabilité principale de cette activité incombe à l'institution où se déroule la recherche ${ }^{18}$.

Dans le texte qui suit, nous nous concentrerons particulièrement sur le rôle du Comité d'éthique de la recherche dans le contexte d'un projet de recherche en génétique. Nous n'examinerons pas la question des audits. Dans notre analyse, nous adopterons une approche globale et emploierons généralement le terme «suivi de l'éthique» afin de désigner globalement les activités du comité d'éthique qui consistent à s'assurer que la sécurité des participants et le respect des règles éthiques demeurent tout au long du déroulement d'un projet de recherche (portant ainsi notre attention tant sur les activités d'évaluation continue que de surveillance). Par ailleurs, le terme «surveillance» sera employé pour désigner plus généralement toutes autres activités entreprises par l'un ou l'autre des acteurs impliqués dans la recherche en vue de veiller au bon déroulement du projet.

15. Bonnes pratiques cliniques, supra note 3., art. 1.6.

16. Ibid., art. 5.19.2 (a).

17. Groupe de travail, supra note 7, à la p. 7.

18. Ibid. 


\section{Nécessité d'assurer un suivi de l'éthique et bilan}

Pour juger de la nécessité pour le comité d'éthique de la recherche d'effectuer un suivi de l'éthique des projets de recherche ou même de s'impliquer dans la surveillance éthique continue, il faut retourner aux sources et examiner son mandat. La définition retenue dans la nouvelle réglementation fédérale en matière d'approbation de nouveaux médicaments offre une bonne vue d'ensemble de ce mandat :

Son principal mandat est d'approuver la tenue de projets de recherche biomédicale sur des sujets humains et d'en contrôler périodiquement le déroulement afin d'assurer la protection des droits des sujets, ainsi que leur sûreté et leur bien-être ${ }^{19}$.

Or, cette définition donne déjà le ton au rôle du CÉR dans la surveillance en parlant de «contrôle périodique». Par ailleurs, il ressort clairement de cet extrait que le noyau dur du mandat du CÉR est la protection des participants. Pour protéger adéquatement les participants, l'approbation initiale d'un projet de recherche par le CÉR est un excellent point de départ, mais il s'agit d'une étape insuffisante en regard de l'objectif poursuivi.

Un projet de recherche n'est pas statique. Sa justification fondamentale peut, à tout moment, être remise en question par l'acquisition de nouvelles connaissances ou de nouvelles informations. Sa faisabilité peut se heurter à des difficultés une fois sur le terrain. Les données intérimaires peuvent forcer des modifications au protocole. L'équipe de recherche elle-même peut subir des remaniements. Ainsi, l'évaluation faite par le comité d'éthique n'est, en quelque sorte, qu'un cliché du projet à son point de départ. Hormis les cas de déviation au protocole, il est possible que le projet lui-même évolue en cours de route. Au surplus, le chercheur peut avoir pris des engagements à l'égard de la réalisation du projet de recherche qui doivent se concrétiser dans sa mise en œuvre sans quoi le projet n'est plus le même que celui envisagé par le CÉR au jour de son approbation. Il importe dès lors de vérifier si l'analyse des tenants

19. Canada, Règlement modifiant le Règlement sur les aliments et drogues (1024-essais cliniques) D.O.R.S./2001-13, art. C.05.001(a). 
et aboutissants sur lesquels le CÉR a fondé sa décision d'approuver un projet de recherche concordent avec son déroulement dans la réalité.

L'approbation du protocole par le CÉR n'a pas plus de valeur que les faits, les hypothèses ou les prédictions sur lesquels elle s'appuie. S'ils changent, les raisons pour lesquelles un protocole a été jugé éthique deviennent moins valides. Le concept de l'examen continu offre une base sur laquelle des systèmes peuvent être établis pour mieux assurer la protection des sujets de recherche, mieux appuyer l'excellence en recherche, et mieux rendre compte aux sujets de recherche et aux Canadiens ${ }^{20}$.

Le suivi de l'éthique et toute autre activité de surveillance en lien avec l'éthique sont d'autant plus importants que le contexte dans lequel se déroule la recherche a beaucoup changé. Un centre de recherche peut avoir des centaines de projets à mener de front, dont plusieurs sont «multicentres» ou même sous la gouverne de d'autres institutions. La recherche comporte d'importants enjeux scientifiques et financiers. Le financement classique gouvernemental et universitaire a largement été remplacé par des partenariats avec l'industrie. La compétition se fait sentir à tous les niveaux et les besoins de trouver des applications commerciales sont pressants. Tous ces éléments contribuent à l'accélération et à la complexification du processus de recherche.

Les projets de recherche eux-mêmes apportent des questions nouvelles qui demandent une réflexion à long terme. Notamment, la génétique a confronté les CÉR canadiens à des questions urgentes auxquelles ils n'avaient jamais songé. Parallèlement à ce nouveau contexte de recherche, on observe une multiplication rapide des normes éthiques tant nationales qu'internationales gouvernant la recherche. Un suivi de ces développements demande une attention particulière.

Enfin, le suivi et la surveillance des projets de recherche n'ont pas pour seul objectif de maintenir la qualité éthique des projets en cours. Par ces activités, on aspire aussi à assumer une mission éducative auprès des équipes de recherche en s'assurant d'intégrer l'éthique dans la réalité quotidienne du 
déroulement d'un projet de recherche. Il s'agit aussi d'établir un dialogue continu entre l'équipe de recherche et le CÉR. Il permet en outre au CÉR de mieux comprendre les conséquences de ces décisions et d'en tirer des leçons pour les autres projets de recherche à venir.

Malheureusement, on constate que le suivi éthique des projets de recherche est quelque peu déficient. Plusieurs auteurs ont dénoncé cette situation dans la doctrine. Déjà en 1995, Charles Weijer et le «Clinical Trials Research Group» dressaient un bilan négatif des activités de suivi ${ }^{21}$. Au Québec, Marie-Hélène Parizeau observait de grandes lacunes à cet égard ${ }^{22}$. Plus récemment, le Vérificateur général du Québec examinait la gestion des activités de recherche réalisées dans des centres hospitaliers. Le constat général du Vérificateur est que les activités de suivi sont déficientes ${ }^{23}$. Plus précisément, le Vérificateur a pris note des éléments suivants :

Nous avons constaté que la plupart des comités d'éthique n'effectuent aucune surveillance éthique continue même si, dans certains cas, leur mandat le prévoit. Actuellement, les efforts des comités se résument

21. C. Weijer et al., «Monitoring Clinical Research : An Obligation Unfulfilled» (1995) 152:12 Can. Med. Assoc. J. 1973.

22. M.-H. Parizeau, Rapport d'enquête concernant les activités des comités d'éthique clinique et des comités d'éthique de la recherche au Québec, Québec, Publications du Québec, 1999 aux pp. 44 et 45 : «Le suivi des protocoles de recherche pose manifestement des problèmes aux CÉR, même si 28 d'entre eux sur 37 disent effectuer une forme de suivi. Certains CÉR écrivent très honnêtement qu'ils ont un problème d'effectif. En fait, le suivi s'avère plus systématique pour les CÉR ayant approuvé des essais cliniques. Les CÉR prennent note des amendements au protocole et des effets cliniques rapportés par la compagnie. Les autres CÉR vérifient occasionnellement les formulaires de consentement signés et demandent plus régulièrement, semble-t-il, un bref rapport au chercheur responsable concernant la fin de sa recherche. Manifestement, le suivi des protocoles de recherche est difficile en pratique et n'est pas systématique, comte tenu des problèmes qui existent déjà pour faire l'évaluation éthique et scientifique des protocoles de recherche».

23. Vérificateur général, supra note 4 à la p. 82 : «Les centres hospitaliers n’ont pas mis en place tous les mécanismes de gestion de l'éthique appropriés aux activités de recherche qui impliquent des sujets humains. On note, par exemple, que les règles en cette matière soutiennent insuffisamment les activités des chercheurs et ne favorisent pas l'efficience des comités d'éthiques de la recherche lors de l'approbation des projets. Qui plus est, la surveillance éthique continue exercée sur ces projets est déficiente et le soutien accordé aux comités, de même que la formation sur l'éthique dispensée aux acteurs impliqués dans les travaux de recherche, laissent à désirer» (nos caractères gras). 
à approuver les projets avant le début des travaux et à recevoir une fois par année le rapport d'étape du chercheur. Beaucoup d'importance est accordée aux documents présentés par le chercheur et au formulaire de consentement, mais on ne s'assure pas que ses travaux et ceux de son équipe correspondent à ce qui avait été approuvé par le comité. ${ }^{24}$

Bien que la surveillance continue apparaisse comme une étape logique dans le processus visant à protéger les participants à des projets de recherche, sa pleine mise en œuvre demeure encore à réaliser. Pourtant, il existe un fondement non seulement logique, mais bien normatif à ce principe. Nous en examinerons les principaux textes dans la prochaine section.

\section{Fondements normatifs de l'obligation de suivi}

Quand on tente de préciser la source de l'obligation de suivi, il est un peu déroutant d'examiner les différents textes normatifs. Ceux-ci n'offrent pas une conception uniforme de ce qu'est un suivi et l'attribution de l'obligation à l'un ou à l'autre des partenaires de la recherche varie. Au surplus, peu de documents vont jusqu'à proposer des moyens concrets de mise en œuvre du principe de suivi. Voici une recension des principaux documents normatifs.

\section{a) Au niveau international}

Au niveau international, l'article 13 de la Déclaration D'Helsinki suggère que le comité d'éthique a «le droit» de surveiller le déroulement d'un projet. L'obligation repose plutôt sur le chercheur qui doit lui offrir l'information nécessaire pour ce faire ${ }^{25}$.

Les Lignes directrices opérationnelles pour les Comités d'éthique chargés de l'évaluation de la recherche biomédicale de l'Organisation mondiale de la santé consacrent une section entière au suivi. Dans cette perspective, il appartient au CÉR d'établir une procédure de suivi. Par ailleurs, la nécessité de

24. Vérificateur général, Rapport, supra note 4, sec. 4.123 à la p. 99.

25. Association médicale mondiale, Déclaration d'Helsinki, Édimbourg, 2000, sec. $13: \ll[\ldots]$ The committee has the right to monitor ongoing trials. The researcher has the obligation to provide monitoring information to the committee, especially any serious adverse events.» 
faire un suivi s'étend du moment où une décision est prise par le CÉR jusqu'à la fin de la recherche ${ }^{26}$. Ces lignes directrices font une liste d'éléments qui doivent être pris en compte dans le suivi des projets de recherche. Certains se sont imposés dans la pratique et sont devenus des incontournables :

Les exemples ou événements suivants imposent un contrôle de suivi d'une recherche :

a. Tout amendement au protocole susceptible d'affecter les droits, la sécurité et/ou le bien-être des participants, ou la conduite de la recherche;

b. Les événements indésirables graves ou inattendus liés à la conduite de la recherche ou au produit testé; et les mesures prises par les investigateurs, le promoteur et les organismes réglementaires;

c. Tout événement ou information nouvelle susceptible de modifier le rapport bénéfice/risque de la recherche ${ }^{27}$. (nos italiques)

\section{b) Au niveau national et provincial}

Au niveau national, les récentes modifications à la Loi sur les aliments et drogues sont sans équivoque quant à la responsabilité du CÉR en matière de suivi :

Comité d'éthique de la recherche $-[\ldots]$

a) son principal mandat est d'approuver la tenue de projets de recherche biomédicale sur des sujets humains et d'en contrôler périodiquement le déroulement afin d'assurer la protection des droits des sujets, ainsi que leur sûreté et leur bien-être ${ }^{28}$. (nos italiques)

26. Organisation mondiale de la santé, Lignes directrices opérationnelles pour les comités d'éthique chargés de l'évaluation de la recherche biomédicale, Genève, 2000, art. 9: «Les CE doivent établir une procédure de suivi pour suivre de (sic) l'évolution de toutes les recherches ayant fait l'objet d'une décision favorable, depuis la date où la décision a été prise jusqu'à la fin de la recherche. Les moyens de communication en cours entre les CE et le demandeur doivent être clairement spécifiés. [...]» [Organisation Mondiale de la Santé, Lignes directrices].

27. Ibid, art. 9.3.

28. Loi sur les aliments et drogues, L.R.C. 1985, c. F-27, art. C.05.001. 
Par ailleurs, les Bonnes pratiques cliniques apportent des précisions quant aux moyens à mettre en place pour effectuer un suivi adéquat dans le domaine pharmaceutique. Elles privilégient un système de rapports à différents organismes, notamment le CÉR.

Tout d'abord, elles incitent le CÉR à mettre sa procédure par écrit. À cet effet, elles dressent une liste des informations que le CÉR devrait exiger du chercheur en cours de projet ${ }^{29}$. Au surplus, l'investigateur doit présenter des résumés écrits faisant état du déroulement de l'essai au CÉR au moins une fois par année ou plus souvent selon les exigences du CÉR ${ }^{30}$. Paradoxalement, les Bonnes pratiques cliniques contiennent une section intitulée «Rapport sur la sécurité» où tous les rapports doivent être obligatoirement remis au promoteur et ce n'est qu'à la demande du CÉR que ces documents lui seront remis ${ }^{31}$.

Il faut noter que le promoteur a une obligation de faire rapport aux investigateurs/établissements et aux CÉR «s'il y a lieu» des réactions indésirables à un médicament qui sont à la fois graves et inattendues ${ }^{32}$. Par

29. Il faut noter que ces éléments ne sont pas des obligations. Il appartient aux CÉR de les inclure dans sa politique. En contraste, l'article 4.11.1 oblige les chercheurs à informer les promoteurs des incidents thérapeutiques graves. Les éléments incluent notamment : «a. des variantes ou modifications apportées au protocole pour éliminer des dangers immédiats pour les sujets participants à l'essai (voir 3.3.7, 4.5.2,et 4.5.4); b. des modifications entraînant un accroissement des risques pour les sujets ou ayant une incidence considérable sur le déroulement de l'essai (voir 4.10.2); c. de toute réaction indésirable à un médicament (RIM) qui est grave et inattendue; d. des nouveaux renseignements sur des éléments susceptibles de nuire à la sécurité des sujets ou au déroulement de l'essai», Bonnes pratiques cliniques, supra note 3, art. 3.3.8.

30. Ibid., art. 4.10.1.

31. Ibid., art. 4.11.1 et 4.11.3: «4.11.1 Tous les incidents thérapeutiques graves (ITG) doivent être signalés immédiatement au promoteur, sauf ceux qui, selon le protocole ou un autre document (brochure de l'investigateur, p. ex.), n'ont pas besoin d'être signalés de toute urgence. Ces rapports urgents doivent être suivis rapidement par des rapports détaillés écrits. Les rapports urgents et les rapports de suivi doivent renvoyer aux sujets uniquement par leurs numéros de code uniques plutôt que par leur nom, leur numéro d'identification personnel ou leur adresse. L'investigateur doit également respecter les exigences réglementaires applicables concernant l'obligation de signaler, aux organismes de réglementation et au CEE/CEI, les réactions indésirables graves à un médicament. [...] 4.11.3 En ce qui concerne les décès, l'investigateur doit fournir au promoteur et au CEE/CEI toute information additionnelle requise (rapports d'autopsie et rapports médicaux finals, p. ex.)». 
ailleurs, le CÉR et l'établissement où se déroule la recherche doivent être informés par le chercheur des abandons ou arrêts prématurés d'étude ${ }^{33}$. Il en va de même lorsque la décision provient du promoteur lui-même ${ }^{34}$. À la fin de l'étude, le CÉR doit recevoir un rapport final au sujet du projet. L'institution peut aussi recevoir ce rapport à sa demande ${ }^{35}$.

Selon les Bonnes pratiques cliniques, il existe aussi une obligation pour le promoteur de mettre en place une surveillance adéquate et des audits s'il y a lieu $^{36}$. Enfin, on indique que le promoteur doit veiller à ce que le participant autorise l'accès au dossier pour des fins de surveillance et de vérification ${ }^{37}$. On s'assure donc de se doter des autorisations requises pour que la surveillance puisse s'effectuer sans enfreindre les règles sur la confidentialité des données de recherche.

Si l'on peut se réjouir des précisions fournies dans les Bonnes pratiques cliniques, il faut toutefois se rappeler que ces règles sont d'application restreinte. Elles ne s'appliquent qu'à l'égard de la recherche menée sur des produits pharmaceutiques et non aux projets de recherche en génétique.

Pour sa part, L'Énoncé de politiques des trois Conseils fait figure de proue, au Canada, en matière de recherche biomédicale. On y réitère la nécessité d'effectuer une surveillance éthique continue. Cependant, l'énoncé est surprenant à deux égards. Premièrement, il confie au chercheur la responsabilité de proposer au CÉR des méthodes de surveillance appropriées à un projet ${ }^{38}$. Deuxièmement, mise à part la surveillance des rapports annuels, il propose que

33. Ibid., art. 4.12.

34. Ibid., art. 5.5.9.

35. Ibid., art. 4.13 .

36. Ibid., art. 5.18 et 5.19 .

37. Ibid., art. 5.15.1.

38. Bien que la collaboration des chercheurs dès le départ soit souhaitable, il aurait peut-être été préférable de dire que les méthodes de suivi sont décidées de concert avec les chercheurs impliqués. En pratique, il est très rare, voire exceptionnel, que les chercheurs aillent d'emblée proposer aux CÉR des méthodes de suivi éthique. 
la surveillance éthique continue ne devrait pas être assumée par le CER ${ }^{39}$. Les trois Conseils recommandent plutôt que les autres partenaires de la recherche s'en chargent. La surveillance y est perçue comme «une responsabilité collective, assumée par tous dans l'intérêt commun de maintenir des critères éthiques et scientifiques irréprochables ${ }^{40}$. Paradoxalement, selon le chapitre sur les essais cliniques (chapitre 7) de l'Énoncé de politiques des trois Conseils, il incombe au CÉR de suivre de façon «stricte et continue» les essais de phase I, pour lesquels une vigilance accrue est indiquée ${ }^{41}$. On peut donc dire que le rôle du CÉR dans la surveillance éthique continue n'y est pas très bien balisé.

L'Énoncé de politique suggère des thèmes sur lesquels devrait porter le suivi tels que l'examen formel du processus de consentement libre et éclairé, la création d'un comité de protection des sujets, l'examen périodique par de tierces personnes des documents générés par la recherche, l'analyse des rapports d'événements externes défavorables et la vérification des dossiers des participants. $^{42}$

Un concept important retenu par les trois Conseils est la notion de proportionnalité des méthodes de suivi en regard du risque que comporte le projet. Le minimum exigé en terme de suivi (lorsque le risque est plus que minimal) semble être les différents rapports d'étape. Par ailleurs, les trois Conseils reconnaissent explicitement qu'il peut être nécessaire de prévoir des méthodes particulières de surveillance «pour faire face à des cas particuliers».

39. Énoncé de politique des trois Conseils, supra note 14, règle 1.13: «a) Toute recherche en cours devra faire l'objet d'une surveillance éthique continue, dont la rigueur devrait être conforme à la méthode proportionnelle d'évaluation éthique. b) Les chercheurs qui soumettent des propositions à des CÉR suggéreront simultanément une méthode de surveillance continue appropriée à leur projet. c) En général, les chercheurs remettront au moins aux CÉR un bref rapport annuel. Les CÉR seront rapidement avisés de la fin des projets.

Exception faite de l'examen rigoureux qu'ils doivent faire des rapports annuels, les CÉR ne devraient pas, sauf dans des cas précis où ils pensent être les mieux placés pour intervenir, se charger de la surveillance continue de l'éthique des projets».

40. Ibid. à la p. 1.12 .

41. Ibid., règle 7.1 .

42. Ibid. 
On peut penser que certains projets de recherche en génétique peuvent commander ce genre d'attention.

Au Québec, des articles du Code civil sont consacrés à la recherche biomédicale. Le suivi y est obligatoire seulement pour les comités d'éthique approuvant des projets de recherche avec des mineurs ou des personnes inaptes. $^{43}$ Cela laisse bon nombre de projets de recherche sans suivi «obligatoire». Au surplus, le Code civil du Québec est avare de commentaires en ce qui a trait aux moyens de mettre en œuvre l'obligation de suivi.

Néanmoins, plusieurs rapports québécois ont réitéré de façon plus claire la nécessité d'effectuer un examen continu à l'égard des projets de recherche en général. Le Rapport Deschamps, en 1998, confie une part importante de la responsabilité d'établir un plan de suivi au CÉR. Le suivi s'inscrit dans la mission du comité d'éthique d'assurer la sécurité des participants.

En outre, ayant à s' assurer notamment de la sécurité des personnes qui participent à des projets de recherche, les comités d'éthique ne doivent pas simplement attendre les résultats finaux de la recherche. D'autant moins qu'à l'heure actuelle, suivant les réponses obtenues au questionnaire, la remise d'un rapport final n'est pas une exigence formelle. Les membres du comité d'éthique doivent ainsi identifier les éléments du suivi du projet incluant la fréquence des rapports qui doivent être soumis, les événements qui doivent être rapportés immédiatement et ceux qui doivent être rapportés régulièrement. ${ }^{44}$

43. Art. 21 C.c.Q «Un mineur ou un majeur inapte ne peut être soumis à une expérimentation qui comporte un risque sérieux pour sa santé [...] Une telle expérimentation doit s'inscrire dans un projet de recherche approuvé et suivi par un comité d'éthique».

44. Québec, Ministère de la santé et des services sociaux, Rapport sur l'évaluation des mécanismes de contrôle en matière de recherche clinique au Québec, Québec, Publications du Québec, 1995 à la p. 109 [Rapport Deschamps]. Aussi, les recommandations suivantes étaient énoncées en page 139 : «75. Le comité d'éthique doit identifier les exigences du suivi requis en ce qui concerne notamment la fréquence des rapports et la divulgation des événements indésirables. 76. Lorsque le volume d'activités de recherche le justifie, une ou plusieurs personnes doivent être désignées pour assurer le suivi des projets de recherche, notamment pour s'assurer de la qualité de l'acte de recherche et de sa conformité avec les décisions découlant de l'évaluation éthique et de l'évaluation financière de même que du respect global du protocole de recherche. [...] 78. La personne responsable du suivi doit, par 
En 1998, le Plan d'action ministériel en éthique de la recherche et en intégrité scientifique du Ministère de la santé et des services sociaux du Québec confiait la tâche aux CÉR de concevoir et mettre en place des mécanismes de suivi pour les projets de recherche en cours.

Les CÉR constituent la pierre angulaire du présent plan d'action. Ils ont la responsabilité d'évaluer la conformité des projets de recherche aux règles éthiques, d'en assurer le suivi éthique, et de veiller à la protection des personnes. ${ }^{45}$

D'ailleurs, selon le plan d'action ministériel, les CÉR devaient se doter de mécanismes de suivi éthique pour les projets de recherche en cours pour décembre $1998^{46}$.

Notons que les organismes de régulation au Canada et aux États-Unis, les entreprises pharmaceutiques et certains organismes subventionnaires effectuent des vérifications afin de s'assurer du respect du protocole de recherche et de l'exactitude des données de recherche déclarées ${ }^{47}$. Cependant, bien qu'il existe un certain chevauchement des activités, il ne faut pas se satisfaire strictement des activités de surveillance scientifique pour couvrir tous les aspects requis par la surveillance éthique d'un projet.

voie d'échantillonnage, vérifier le déroulement des projets de recherche, la qualité du processus d'obtention et de collecte des données, ainsi que la qualité du consentement éclairé et de la nature des inconvénients encourus par les personnes participants au projet de recherche. [...] 80. Le comité d'éthique doit recevoir du chercheur un rapport annuel lui indiquant ce qu'il est advenu du projet de recherche.»

45. Québec, Ministère de la santé et des services sociaux, Plan d'action ministériel en éthique de la recherche et en intégrité scientifique, Québec, Publications du Québec, 1998 à la p. 13 [Ministère de la santé et des services sociaux, Plan d'action].

46. Ibid. à la p. 14 .

47. Vérificateur général, Rapport, supra note 4 à la p. 99. 


\section{Conclusion de la Partie I}

On peut résumer ainsi les grandes lignes qui semblent sous-tendre l'obligation d'effectuer un suivi éthique des projets de recherche : (1) s'assurer du maintien des conditions propices au déroulement éthique du projet de recherche (incluant la protection des participants) et qui ont initialement donné lieu à son approbation; (2) s'assurer de la pleine mise en œuvre des différents engagements pris par le chercheur; (3) identifier les problèmes éthiques soulevés en cours de déroulement de projet et évaluer les ajustements requis en cours de projet.

Pour ce faire, le suivi doit porter sur diverses dimensions d'un projet de recherche. Il faut porter attention à plusieurs niveaux, dont, notamment, le respect du participant, le respect des principes éthiques directeurs, l'adhésion au protocole de recherche, le respect du processus d'approbation éthique lui-même, etc. ${ }^{48}$.

Compte tenu de l'obligation d'assurer une surveillance éthique continue, il est impératif de se doter de différents outils pour pouvoir réaliser le suivi des projets en cours de recherche. Certains mécanismes, visant essentiellement à tenir le CÉR informé, semblent faire l'unanimité dans les textes normatifs. Ainsi, l'obligation de soumettre un rapport annuel au CÉR ${ }^{49}$ et un rapport de fin de projet ${ }^{50}$ sont réellement obligatoires pour tous les projets, et au premier chef pour les projets en pharmaceutique. Si le risque est plus que minimal, l'Énoncé de politique des trois Conseils requiert aussi que des rapports d'étape soient produits. En outre, les Bonnes pratiques cliniques rendent obligatoire de faire rapport au CÉR de toute suspension ou arrêt de l'étude par l'investigateur ou le

48. Il faut noter que la surveillance des aspects scientifiques d'un projet est assumée en partie par d'autres organismes, par exemple un DSMB. Cependant, bien que la surveillance effectuée par cet organisme soit pertinente pour le suivi que doit effectuer le CÉR, elle ne saurait le remplacer. En effet, les préoccupations purement éthiques du CÉR ne sont que partiellement servies par un rapport de DSMB.

49. Énoncé de politique, supra note 14, art. 1.13c); Bonnes pratiques cliniques, supra note 3, art. 4.10.1.

50. Énoncé de politique, ibid., art. 1.13 c) in fine; Bonnes pratiques cliniques, ibid., art. 4.13. Il faut noter que l'Énoncé de politique suggère une simple notification plutôt qu'un rapport. 
promoteur $^{51}$. Cependant, rappelons que les Bonnes pratiques cliniques ne sont obligatoires qu'à l'égard des projets de recherche en pharmaceutique. De plus, ces obligations ne sont que des moyens minimaux à mettre en oeuvre.

D'autres mécanismes sont laissés aux initiatives locales et doivent jouir d'une certaine flexibilité pour pouvoir se moduler en fonction du niveau de risque et de complexité d'un projet de recherche. Les comités sont fortement encouragés à se doter de lignes directrices plus précises à l'égard du suivi ${ }^{52}$. De plus, ils doivent élaborer, en collaboration avec les chercheurs, des plans de suivi des projets qu'ils approuvent. Ces plans peuvent être adaptés au contexte particulier de recherche, notamment celui de la génétique.

Quel serait un plan de suivi approprié à la recherche en génétique? Après ce tour d'horizon de l'obligation de surveillance éthique continue et des mécanismes pour la mettre en œuvre, examinons maintenant les besoins particuliers de la génétique.

\section{Partie II : Le suivi de l'éthique dans le contexte particulier de la recherche en génétique humaine}

«The problem for ethics committees is that they are faced with these difficult questions now, and that in contrast with well established notions of, for example, individual privacy and autonomy, the whole field of genetic research is so new and is moving so fast that guiding landmarks are very few. [...] Thus, current systems of ethical oversight are lagging well behind the capabilities of science and frequently it is left to individual investigators to set and follow rules for their work. $\rangle^{53}$

51. Bonnes pratiques cliniques, supra note 3, art. 4.12.1 et 4.12.3.

52. Voir notamment ibid., art. 3.3.8 et 4.11.1; Rapport Deschamps, supra note 44 à la p. 109; Ministère de la santé et des services sociaux, Plan d'action, supra note 45 à la p. 14; Organisation Mondiale de la Santé, Lignes directrices, supra note 26, sec. 9.

53. K. Jamrozik, «The Case for a New System for Oversight of Research on Human Subjects» (2000) 26 Journal of Medical Ethics 334 à la p. 338. 
Alors que les CÉR et la communauté scientifique sont encore en train d'apprivoiser les problématiques soulevées lors de l'évaluation de projets de recherche en génétique, voilà que surgit un autre problème de taille : le suivi de l'éthique de ces projets. Dans le cadre des activités du CÉR, la surveillance éthique continue comporte des lacunes que l'on reconnaît d'emblée. Au surplus, le suivi des projets de recherche en génétique comporte des difficultés supplémentaires.

La nature des projets de recherche en génétique est fort différente des projets de recherche «traditionnels» tels qu' on les envisage en pharmaceutique. Qu'il s'agisse de la participation des individus, du type de données recueillies ou des besoins en terme de recherche, les stratégies de recherche sont envisagées sous un angle différent. Cela nous force à reconsidérer la nature et l'étendue de l'obligation de suivi. Est-ce que l'obligation de suivi continue à s'appliquer? Par ailleurs, beaucoup de recherches se déroulent dans plusieurs centres à la fois. La nature même de la recherche en génétique nécessite d'avoir accès à un nombre d'échantillons si important qu'il faut souvent dépasser les murs d'une seule institution ou même mettre en place des «méga-banques» de tissus. L'échange de données est courant et nécessaire. Cela rend le suivi encore plus complexe.

Compte tenu de ce contexte assez particulier, plusieurs questions se posent. Comment assurer un suivi dans un tel contexte? Au-delà des problèmes logistiques qu'un tel suivi peut représenter, qui doit en prendre la charge? Jusqu'où va la responsabilité des divers intervenants à l'égard du suivi? Quels sont les engagements réels que nous sommes en mesure d'assumer en matière de suivi de l'éthique des projets de recherche en génétique?

Le suivi de l'éthique des projets de recherche en génétique est truffé de difficultés au point de faire douter de la capacité des CÉR à mener à bien une telle entreprise. Nous tenterons d'apporter au moins des éléments de réflexion à ces questions. Dans le document qui suit, nous discuterons premièrement de la pertinence de la surveillance continue en génétique humaine. Deuxièmement, nous identifierons le point de mire approprié du suivi en génétique humaine. Troisièmement, nous aborderons les difficultés particulières posées par la recherche «multicentrique» en génétique. Enfin, nous formulerons des 
recommandations d'ordre pratique pour le suivi éthique de la recherche institutionnelle en génétique.

\section{La pertinence d'un suivi et d'une surveillance éthique dans la recherche en génétique humaine}

En préambule, il est nécessaire de valider que la recherche sur l'ADN doit faire l'objet d'un suivi éthique. Comme on le constate, le suivi porte une attention spéciale à la protection des sujets humains. Or, compte tenu que le risque pour l'intégrité des participants et leur bien-être physique est plutôt limité dans le cas d'un projet de recherche en génétique, est-il nécessaire d'assurer un suivi? Ne pourrait-on pas conclure que l'échantillon d'ADN est «donné» pour la recherche dans des circonstances qui sont évaluées par un CÉR et, pourvu que ces circonstances apparaissent acceptables, qu'il n'y a pas lieu de poursuivre le travail du CÉR plus loin?

Pour répondre à cette question, nous répondrons tout d'abord à la question de savoir si la recherche sur l'ADN, une fois détaché du corps d'un individu, constitue de la recherche sur des sujets humains au sens des différents textes normatifs? L'Énoncé de politique des trois conseils est clair à ce sujet ${ }^{54}$. Le Code civil n'a pas le mérite d'être aussi clair ${ }^{55}$. On peut cependant examiner les textes normatifs internationaux pour s'en convaincre ${ }^{56}$. Compte tenu que nous croyons que la recherche sur l'ADN constitue bel et bien de la recherche avec des êtres humains, on peut donc conclure que l'obligation de suivi doit s'exercer même dans le cas d'un projet de recherche en génétique.

Au demeurant, la protection du participant n'est pas qu'une question d'intégrité physique. On doit aussi porter attention à d'autres questions telles que la protection de la vie privée ou de la confidentialité qui sont des sujets

54. Voir la définition de recherche dans Énoncé de politique, supra note 14, règle 1.1.

55. Bien que le Code civil soit généralement interprété en ce sens, l'expérimentation n'y est pas clairement définie. Le fait que l'ADN soit détaché de la personne pour la recherche pose des défis conceptuels particuliers. Puisqu'il s'agit de recherches avec des tissus humains, on conclut généralement qu'il s'agit de recherches sur des sujets humains. De toute façon, l'étape du prélèvement de tissus constitue clairement une atteinte à l'intégrité de la personne pour laquelle un consentement est requis.

56. Voir par ex. UNESCO, Déclaration Universelle sur le génome humain, Paris, 1997, art 5. 
chauds en matière de génétique. Le suivi ne porte pas que sur la protection du participant. Il a pour but de s'assurer que le projet, de façon générale, se déroule de façon éthique. Le suivi de l'éthique des projets de recherche en génétique apparaît donc non seulement approprié mais impératif.

Par ailleurs, une banque d'ADN doit-elle, en elle-même, faire l'objet d'un suivi éthique? En effet, tous les projets de recherche en génétique nécessitent la mise en banque d'ADN ou de tissus à court ou à long terme. On peut imaginer qu'une banque d'ADN continue d'exister après la fin du projet de recherche pour lequel elle a été mise sur pied. Nous croyons que dans la mesure où une banque est créée en vue de faire de la recherche, il s'agit d'une activité indissociable de tout projet de recherche qui nécessite un suivi éthique. Le suivi doit donc porter non seulement sur la réalisation du projet lui-même, mais aussi sur le maintien de la banque qui rend ou a rendu la recherche possible. Il est certes souhaitable que le CÉR joue un rôle dans le caractère éthique de l'utilisation des banques constituées à des fins de recherche. Son rôle peut s'avérer particulièrement important dans les périodes de transition ou les fins de projet afin d'éviter que la banque d'ADN ne sombre dans l'oubli. Nous verrons dans la section 3 que la question est beaucoup plus complexe dans le cas d'une banque d'ADN conservée à l'extérieur de l'institution où siège le CÉR.

Le suivi en génétique aura donc deux pôles importants d'attention : le projet de recherche lui-même et la banque d'ADN qui permet sa réalisation.

\section{Fixer le point de mire du suivi de l' éthique des projets de recherche en génétique}

Dans le domaine de la recherche clinique pharmaceutique, le participant est un collaborateur à part entière. Il prête non seulement son corps à la science, en acceptant d'ingérer un médicament pour qu'on en observe les effets biologiques, mais aussi sa collaboration pleine et entière, par exemple, en se rendant aux différents rendez-vous de suivi, en se soumettant à des examens médicaux pour mesurer les effets de la molécule, etc. C'est véritablement son intégrité physique qu'il met au service de la science. Ce faisant, il s'expose évidemment à des risques de nature physique. Ainsi, le médicament peut en 
effet avoir des effets secondaires inattendus et indésirables qu'il faut surveiller étroitement. Au surplus, il est également possible qu'on exerce des pressions pour qu'il continue de collaborer en se présentant aux rendez-vous, l'accumulation d'informations au sujet de son évolution clinique étant essentielle dans une démarche scientifique visant à démontrer l'efficacité et l'innocuité d'un produit pharmaceutique.

Dans un tel contexte, il n'est pas étonnant que les mécanismes de surveillance soient axés sur la protection physique des participants et le respect de leur personne. On veut s'assurer qu' on veille adéquatement à leur bien-être, que tous les risques sont minimisés et que leur participation s'explique par leur volonté seule de prêter leur concours et non en raison de coercitions ou pressions indues.

En matière de recherche en génétique, le contexte est quelque peu différent, ce qui change les besoins en terme de suivi. Comme il a été maintes fois réitéré dans la littérature, les risques liés à la recherche en génétique sont souvent d'une autre nature que purement physique ${ }^{57}$. Dans le domaine de la recherche en génétique, il est fort possible que la «participation active» du sujet se limite à une prise de sang combinée avec un suivi de l'évolution médicale qui peut parfois se faire sur un simple examen du dossier médical. Par exemple, pour des recherches visant à établir un test génétique, bien que certaines données du dossier médical soient habituellement requises pour surveiller l'évolution clinique d'un patient, des examens ou procédures supplémentaires ne sont pas toujours nécessaires ${ }^{58}$. Le bien-être physique du participant n'est pas mis en cause par la procédure expérimentale ${ }^{59}$ de la même façon que dans un projet pharmaceutique. Il faut certes se préoccuper de la façon dont on obtient les échantillons sanguins nécessaires aux analyses d'ADN. Les préoccupations

57. G. Cardinal et al., «Recherche en génétique humaine et consentement» Fonds de recherche en santé du Québec (FRSQ) et Réseau de médecine génétique appliquée (RMGA) 2000. Aussi disponible en ligne : Réseau de Médecine Génétique Appliquée, http://www.rmga. qc.ca.

58. Le domaine de la pharmacogénomique fait évidemment exception à cette règle.

59. L'auteure ne suggère pas que l'intégrité physique du participant n'est pas importante en recherche génétique. Il faut évidemment protéger les participants contre tout abus en matière de recrutement. Cependant, une fois la prise de sang effectuée, la participation active du participant est limitée. 
liées à la liberté de participation et au consentement véritablement éclairé demeurent à l'égard de l'échantillonnage sanguin. Cependant, on se rend bien compte que les questions de l'intégrité de la personne et de la sécurité physique de celle-ci sont plus limitées. La recherche peut se dérouler sans une implication active du participant. En fait, techniquement, elle peut même se dérouler complètement à son insu. L'ADN étant détaché de son corps, il est à la fois messager de son titulaire de par l'information qu'il porte et physiquement séparé de ce dernier ${ }^{60}$.

L'intégrité physique n'est donc peut-être pas la cible appropriée de surveillance dans la recherche en génomique. Sans l'exclure, il faut porter notre attention sur d'autres aspects de la recherche. Une réflexion sur notre approche du suivi éthique des projets de recherche est requise. Quels sont donc les éléments qui demandent une attention particulière dans le domaine de la surveillance des projets de recherche approuvés par un comité d'éthique à la recherche et des banques d'ADN qui sont constituées dans un tel cadre? Les recommandations énumérées en guise de conclusion tentent de cibler les secteurs qui requièrent une attention accrue de même que les mécanismes à mettre en place pour leur assurer une surveillance adéquate.

D'entrée de jeu, on peut déjà anticiper l'importance de thèmes tels que la protection de la vie privée et de la dignité du participant. Plus particulièrement, on doit porter une attention toute spéciale aux mécanismes de protection des données des participants, au processus de communication des résultats personnels, au protocole de diffusion des résultats et, dans une plus large perspective, au respect des politiques de gestion de la banque d'ADN. On doit aussi se pencher sur le respect de la volonté du participant à l'égard de l'échantillon et une utilisation de celui-ci qui soit conforme à l'ordre moral. En terminant, il est utile de rappeler que les mesures déployées pour effectuer un suivi de l'éthique devront être proportionnelles aux risques encourus par les participants.

60. Voir à ce sujet R. P. Kouri et S. Philips-Nootens, Le corps humain, l'inviolabilité de la personne et le consentement aux soins : Le regard du législateur et des tribunaux civils, Sherbrooke, Éditions R.D.U.S., 1999 au chapitre 2. 


\section{Un cas particulièrement difficile : La recherche «multicentre» en génétique ou effectuée à partir d'une banque externe}

Les recommandations qui seront présentées dans la section suivante prennent pour hypothèse que le projet de recherche et la banque de d'ADN sont situés dans la même institution que le CÉR qui approuve le projet. Toutefois, les projets de recherche en génétique nécessitent souvent la création d'une banque d'ADN incluant des échantillons provenant de différentes institutions. Qu'advient-il alors si les tissus sont acheminés à l'extérieur de l'institution? On peut penser que cette banque peut servir au projet de recherche initialement approuvé (ex. : projet de recherche «multicentre») ou même à d'autres projets de recherche. Cette banque peut dès lors se trouver dans une autre ville, voire un autre pays. Elle peut être alimentée par une ou plusieurs autres institutions.

Cela pose des défis de taille dans le domaine du suivi éthique. Le CÉR qui approuve l'envoi de l'échantillon à l'extérieur de l'institution conserve-t-il une obligation de suivi à l'égard de ces échantillons? Cette question nous force à préciser l'étendue de l'obligation de suivi. Nous croyons que le suivi se rattache d'abord et avant tout à l'approbation d'un projet de recherche relevant de sa compétence. Donc, même si l'échantillon quitte l'institution, il nous semble qu'il demeure une obligation d'en faire le suivi dans le cadre du projet de recherche approuvé. Il faut donc demander des rapports et mettre en œuvre toutes autres mesures de suivi éthique jugées utiles, comme s'il s'agissait d'une banque au sein de sa propre institution.

Toutefois, si la banque (externe) est utilisée à d'autres fins que le projet de recherche initial examiné par le CÉR local, la situation se complique. Il ne s'agit pas de se demander si un suivi est pertinent mais plutôt s'il appartient encore au CÉR local de l'exercer et surtout s'il est en mesure de le faire. On pourrait bien entendu envisager une collaboration ou une délégation du suivi de l'éthique à un CÉR plus «près» des activités de cette banque. Toutefois, la question demeure entière pour des banques détenues par des entités qui ne font que prêter le matériel à d'autres chercheurs. Ces banques, de type «fournisseur», ne sont pas nécessairement créées dans le but d'un projet précis, 
mais plutôt en vue d'offrir à d'éventuels chercheurs du matériel de recherche ${ }^{61}$. Dans ce cas, il n'est pas certain qu'il existe un CÉR «central» pour la banque : toutes les activités éthiques sont peut-être décentralisées et s'articulent autour des différents projets de recherche qui utilisent la banque ${ }^{62}$. Qu'en est-il des banques d'ADN détenues par des entreprises privées mais pour lesquelles le prélèvement s'est effectué dans un hôpital du réseau de la santé? Que faire avec des banques «dites orphelines» ou devenues désuètes? Qui en assume alors le suivi éthique? Il est difficile de savoir jusqu'où va l'obligation de suivi éthique pour les CÉR des différentes institutions québécoises et qui peut réellement l'assumer dans de telles circonstances. On peut affirmer qu'on observe un décalage entre les attentes en matière de suivi éthique du projet de recherche et la réalité.

Alors que l'obligation de suivi éthique s'applique à tous les projets de recherche approuvés par un CÉR, comment peut-on assumer une telle obligation en pratique? Quelle que soit l'étendue de l'obligation de suivi, il demeure trois problèmes d'envergure : (a) un problème de contrôle et d'information, (b) un problème d'encadrement normatif et, enfin, un (c) problème de juridiction et de sanction. Il convient ici de cerner les limites du travail que le CÉR local est à même d'effectuer et de faire connaître ces limites aux participants. Nous examinerons donc tour à tour les difficultés auxquelles font face les CÉR.

61. Certains projets d'envergure (privés ou publics) visent la création de banques d'ADN, sans vraiment les rattacher nommément à un projet de recherche. Ces banques sont ni plus, ni moins que des points de services pour les chercheurs. Dans ce cas, il est difficile d'identifier une entité qui sera responsable du suivi. Évidemment, toute utilisation de la banque donnera lieu à l'approbation par un CÉR, mais on peut difficilement envisager qu'une multitude de CÉR de toutes les institutions d'où proviennent les échantillons puissent assumer ce genre de suivi. Il y a donc lieu de réfléchir à la création d'entités de surveillance qui sont au-delà des institutions locales et qui assument ou coordonnent ce genre de suivi pour les institutions locales (On peut imaginer que les CÉR locaux pourraient obtenir rapport ou encore déléguer carrément le suivi à cette autre entité). Ces «super-CÉR» pourraient d'ailleurs simplifier la tâche des CÉR locaux. Sans remplacer les CÉR locaux, ceux-ci pourraient déléguer en tout ou en partie, leur devoir de surveillance à une entité mieux placée pour effectuer le suivi. Le défi est alors de maintenir l'indépendance de cette entité et d'assurer un respect des désirs exprimés par les CÉR locaux qui ont autorisé le transfert d'échantillons à la banque.

62. Cela n'exclut nullement la possibilité que l'entité responsable de la banque (privée ou autre) confie le suivi éthique à long terme à un organisme indépendant. 


\section{a) Un problème de contrôle et d'information}

Premièrement, lorsqu'un CÉR évalue les conditions de mise en place d'une banque qui se déroule dans sa propre institution, il connaît la personne qui en assumera la responsabilité, le contexte général de l'institution et même les lieux où se déroulera la recherche (à tout le moins, il est possible de les visiter au besoin). En revanche, lorsque les échantillons vont à l'extérieur de l'institution, voire à l'étranger, il est nettement plus difficile de juger de la situation réelle qui prévaut dans l'institution détentrice de la banque et en cas de doute, la visite des lieux est rarement une option réaliste. Le CÉR doit se fier aux seules affirmations écrites du chercheur. Dans ce contexte, la cueillette d'information écrite et de qualité est fondamentale. Les renseignements fournis doivent être précis et énoncer clairement qui sera responsable de la banque, où la banque est conservée, les mesures de sécurité mises en place, la politique de mise en banque et l'utilisation envisagée. Le CÉR ne doit pas hésiter à poser des questions précises.

En outre, il ne suffit pas de fournir des informations mais il faut que cela se traduise par des engagements fermes qui se concrétiseront. Compte tenu qu'il est rarement possible pour le CÉR d'effectuer une surveillance sur le terrain, il est nécessaire que la situation décrite soit fidèle à la réalité et que toute modification relative aux politiques de gestion ou à l'orientation de la banque soit soumise au CÉR.

Dans ce contexte, il est important de formuler un certain nombre d'engagements auxquels la banque externe sera tenue de se conformer. Ces engagements, de nature contractuelle, doivent inclure l'envoi de rapports périodiques au sujet de la banque (gestion, échange ou envoi de tissus ou d'ADN à d'autres banques, destruction, etc.), les conditions précises dans lesquelles seront conservés les échantillons, la procédure pour contacter les participants (le cas échéant) et les utilisations des échantillons (pour le présent et l'avenir). Il est clair que ces engagements ne doivent pas être de nature à entraver la recherche. Cependant, ils doivent aller à l'essence du respect de la personne et du participant et témoigner d'un véritable partenariat avec ceux et celles qui fournissent leur ADN pour faire avancer la science. 
Il faut mentionner que, bien que le formulaire de consentement puisse refléter ces engagements, il ne s' agit pas du bon véhicule pour véritablement lier le banquier. Cela ne ferait que surcharger et «contractualiser» ce document ${ }^{63}$. De plus, compte tenu qu'il s'agit d'un engagement contractuel, il est nécessaire que le chercheur et l'institution participent à cette entreprise et en prennent même l'initiative. Il serait donc approprié de prévoir un document pour énoncer ces engagements.

Les CÉR locaux ne peuvent que veiller à ce que les rapports fournis témoignent d'un déroulement conforme aux engagements pris par la banque et le chercheur responsable de celle-ci. Par exemple, si la banque s'engage à détruire les échantillons à la demande des participants, un rapport d'une telle destruction devrait être envoyé au CÉR. Dans l'état actuel des choses, il est irréaliste d'envisager qu'ils feront un suivi plus serré. Cependant, d'autres entités pourraient peut-être assumer cette tâche ou aider les comités d'éthique dans ce sens. Sans remettre en question les comités d'éthique locaux, il faut peut-être songer à la mise en place d'un comité d'éthique pour des projets d'envergure nationale ou internationale ou même à confier à un organisme le pouvoir de surveiller les renseignements de toutes les banques d'ADN sur le territoire de la province.

\section{b) Un problème d'encadrement normatif}

Un deuxième problème rencontré en matière de suivi de projet de recherche en génétique de nature «multicentrique» a trait au cadre normatif. Lorsqu'un CÉR se penche sur l'approbation d'un projet de recherche, il tient compte du cadre normatif canadien et québécois actuellement en vigueur. De plus, les CÉR étant des entités devant tenir compte des réalités locales, ils considèrent aussi les politiques locales de même que les valeurs et les besoins locaux. Cependant, lorsque les échantillons quittent l'institution pour être conservés dans une banque à l'extérieur, ils sortent du cadre normatif en vigueur

63. On peut observer actuellement une tendance des CÉR à utiliser le formulaire de consentement pour lier la banque. Cette situation s'explique par le fait que le contrat de recherche est souvent déjà négocié lorsque le projet est soumis au CÉR. De toute façon, les CÉR n'ont pas à participer à cette négociation. Le seul outil sur lequel ils peuvent avoir de l'influence est le formulaire de consentement. 
dans l'institution, la province ou même le pays. Les échantillons peuvent dès lors être soumis à des règles d'utilisation parfois fort différentes. Comment surveiller ce qui se fait ailleurs selon des règles avec lesquelles nous ne sommes pas familiers?

Prenons un exemple. Aux États-Unis, sont exclues de l'application du titre 45 les recherches menées sur une collection déjà existante d'échantillons anonymes amassés dans le cadre de soins :

Research involving the collection or study of existing data, documents, records, pathological specimens or diagnostic specimens, if these sources are publicly available or if the information is recorded by the investigator in such a manner that subjects cannot be identified directly or through identifiers linked to the subjects. ${ }^{64}$

Une telle recherche n'est tout simplement pas considérée comme de la recherche sur des sujets humains au sens du Code of Federal Regulation.

Par ailleurs, pour des échantillons déjà amassés dans le cadre de recherches et anonymisés, ceux-ci peuvent faire l'objet d'une procédure accélérée d'approbation de la part du comité d'éthique à la recherche ${ }^{65}$ et il peut être dérogé à l'obligation d'obtenir le consentement des participants ${ }^{66}$. La situation est loin d'être aussi claire au Québec, notamment avec les exigences du Code civil. Dans un tel contexte, comment s'assurer que les engagements pris ici seront respectés ailleurs? Bien que le CÉR puisse demander des comptes au détenteur de la banque, il est difficile de savoir quelles règles y seront appliquées. Comment peut-on alors assurer un suivi éthique du projet de recherche?

Dans l'état actuel des choses, il n'est pas évident pour les comités d'éthique d'avoir à approuver des projets faisant appel à des banques tenues dans d'autres pays. Les CÉR n'ont ni les ressources ni le temps pour faire une

64. Titre 45 Code of Federal Regulation, sub-section 46.101(b) 4). Le Code s'applique à toute recherche financée par une agence fédérale ou réglementée par une agence fédérale.

65. Ibid., sub-section 46.110.

66. Ibid., sub-section 46.117(c). 
analyse en profondeur du cadre juridique s'appliquant en l'espèce. Un engagement ponctuel «inter-institutionnel» de mise en banque peut aider à veiller au respect des points les plus importants existant dans notre cadre juridique ou même faire imposer, par contrat, les règles juridiques en vigueur au Québec.

\section{c) Problème de sanction}

Finalement, le troisième problème est lié aux sanctions. Au Québec, les CÉR qui oeuvrent au sein des centres publics sont rattachés au conseil d'administration de l'institution pour laquelle ils oeuvrent. Leur pouvoir de sanction se rattache directement au cœur de l'institution.

Lorsque le CÉR est rattaché directement au Conseil d'administration d'une institution qui abrite des échantillons, son contrôle est direct et les pouvoirs de sanctions multiples. Lorsque les tissus quittent l'institution pour être conservés dans une banque à l'extérieur, ils sortent non seulement à l'extérieur du cadre normatif institutionnel mais aussi à l'extérieur du contrôle effectif de l'institution et du CÉR local. Dans un tel contexte, quel est son réel pouvoir advenant une situation douteuse? Il est toujours possible de demander au chercheur local de cesser sa participation à l'étude, mais comment exiger de faire revenir ou faire détruire les échantillons ${ }^{67}$ ?

On connaît toute l'importance de formuler clairement les attentes du CÉR et de l'institution participante à l'égard du banquier d'ADN, à plus forte raison lorsque la banque est située à l'extérieur de l'institution. Ce cas d'espèce réaffirme la nécessité de signer des engagements contractuels et/ou de connaître les règles de mise en banque qui régissent les activités du banquier. Ces documents constituent d'autres piliers importants sur lesquels le CÉR peut s'appuyer pour faire reconnaître et exécuter certains engagements.

Par ailleurs, il y a peut-être lieu de faire preuve de transparence à l'égard des participants en reconnaissant, dans certains cas, les limites au suivi qui

67. Notez qu'il ne s'agit pas d'un projet de recherche pharmaceutique donnant application à la loi fédérale. 
pourra être effectué par le CÉR (et l'institution qui l'abrite) au sujet de ces échantillons.

Ces trois sujets démontrent bien toutes les difficultés que rencontrent les CÉR lorsqu'ils examinent des projets de recherche avec mise en banque d'ADN à l'extérieur de leur institution. Face à ces difficultés, il y a lieu de songer à de nouveaux modèles de gouvernance pour ces types de recherche.

\section{Recommandations pratiques pour le suivi de l'éthique des projets de recherche institutionnelle en génétique humaine}

Nous croyons fermement que la surveillance ne doit pas être conçue de façon à constituer une entrave à la recherche. Elle doit toutefois participer au maintien d'un environnement de recherche et de pratiques de recherche favorisant la préservation du lien de confiance avec les participants. Elle doit veiller au respect des règles légales et éthiques régissant la recherche. Voici quelques thèmes à aborder en matière de surveillance éthique continue des projets de recherche en génétique humaine.

\section{a) Prélude à la surveillance : l'identification des banques d'ADN existantes et leurs politiques respectives}

L'Énoncé de politique des trois Conseils indique que toute recherche menée sur des tissus humains nécessite une évaluation éthique ${ }^{68}$. Le plus souvent, c'est via l'élaboration d'un projet de recherche amassant ou utilisant des tissus humains qu'un CÉR sera appelé à se pencher sur la banque dans laquelle ces tissus seront conservés. Malheureusement, on peut observer que la recension des banques d'ADN n'est pas toujours faite dans toutes les institutions qui en hébergent ${ }^{69}$.

Pourtant, la surveillance d'un projet de recherche impliquant la création d'une banque d' ADN suppose évidemment que le CÉR en connaisse l'existence.

68. Énoncé de politique, supra note 14 , règle $1.1 \mathrm{~b}$ ).

69. On doit reconnaître qu'il existe une difficulté quant à l'identification même de ce que constitue une banque d'ADN. Il faudrait peut-être élargir ce souhait pour toutes les biobanques utilisées en recherche. 
Il serait utile que les banques de tissus ou d'ADN qui sont conservées au sein d'une institution fassent l'objet d'un registre permettant d'en relever l'existence et d'en connaître les principaux paramètres (types de tissus et lieu où ils ont été collectés, personne responsable de la banque, date du début et de la fin projetée de l'existence de la banque, financement, etc... $)^{70}$. Cette responsabilité incombe à l'institution et au chercheur responsable du projet de recherche. À ce chapitre, il est intéressant de signaler au passage qu'à l'échelle provinciale, toute personne qui «exploite» une banque de tissus à des fins scientifiques, hors d'un établissement de santé du réseau, doit détenir un permis $^{71}$. Si ces dispositions s'appliquent aux entités privées qui mettent sur pied des banques d'ADN à des fins de recherche, cela permettrait peut-être d'en avoir une liste via les permis qui sont octroyés.

Par ailleurs, la surveillance suppose aussi l'adhésion à un certain nombre de principes ou de règles qui doivent être connus dès le départ. L'encadrement de la collecte, de la gestion et de l'utilisation d'une banque d'ADN est nécessaire. Au-delà des grands principes philosophiques de base qui doivent orienter toutes les actions du gestionnaire de la banque («non-malfaisance», bienveillance, etc.), des règles précises relatives à l'administration de la banque doivent être énoncées et respectées. Les banques d'ADN devraient ainsi faire l'objet d'une politique de gestion écrite permettant d'en comprendre les règles de fonctionnement ${ }^{72}$. Ces règles doivent notamment régir les mesures de

70. Un avis récent de la Commission de l'éthique, de la science et de la technologie recommandait d'ailleurs la création d'un répertoire public, par souci de transparence et pour rendre possible «l'exercice d'activités de surveillance ou de suivi de la protection des intérêts des citoyens». Commission de l'éthique de la science et de la technologie, Les enjeux éthiques des banques d'information génétique : pour un encadrement démocratique et responsable, Gouvernement du Québec, 2003 à la p. 38.

71. Loi sur les laboratoires médicaux, la conservation des organes, des tissus, des gamètes et des embryons et la disposition des cadavres, L.R.Q. c. L-0.2, art. 31 : «À l'exception de l'Institut national de santé publique du Québec, nul ne peut exploiter un laboratoire, une banque d'organes et de tissus, s'il n'est titulaire d'un permis délivré à cette fin par le ministre». Il faut noter que le mot «exploite» réfère à une notion d'exploitation commerciale. Dans ce contexte, nous croyons que peu de banques d'ADN employées en recherche pourraient répondre à cette exigence au Québec. Cependant, compte tenu qu'il n'existe pas vraiment de recension des banques d'ADN au Québec, nous n'en savons rien.

72. C. Laberge et al., Énoncé de principes du Réseau de Médecine Génétique Appliquée, version 2000, en ligne : Réseau de Médecine Génétique Appliquée http://www.rmga.qc.ca, sec. I.2. 
sécurité mises en place pour conserver les échantillons, les règles d'accès et d'utilisation des échantillons, les modalités de destruction des échantillons ou encore la finalité de la banque. La surveillance des recherches effectuées à l'aide d'une banque d'ADN doit s'appuyer sur cette politique.

Si tel n'est pas le cas, les institutions concernées et les CÉR devraient à tout le moins veiller à ce que certains engagements ponctuels soient pris, au départ, via le protocole de recherche ou le contrat de recherche à l'égard des participants et de l'utilisation éthique des échantillons de recherche. Ces engagements, qui témoignent d'une vision à long terme de programmes de recherche, visent à assurer la pérennité de la banque tout en respectant la volonté des participants.

Tous ces engagements doivent se refléter adéquatement dans un formulaire de consentement qui fait état du prélèvement, du projet de recherche et de la mise en banque de l'ADN (s'il y a lieu). Ce document est déterminant dans l'utilisation qui sera faite des échantillons prélevés. Tout échange d'échantillons entre différentes équipes de recherche ou différentes institutions devrait être accompagné du formulaire de consentement qui a permis le prélèvement.

\section{b) Le respect des engagements pris à l'égard de la conservation des échantillons}

Lors de la mise en banque d'ADN pour fins de recherche, le chercheur doit prendre un certain nombre d'engagements relatifs à la bonne conservation de ces échantillons. D'une part, ces engagements visent à préserver la bonne qualité de l'échantillon pour qu'il demeure utile à la recherche (exemple : conserver dans des endroits appropriés, à une température assurant sa conservation, etc.). Le gaspillage des ressources de recherche est évidemment à proscrire. Il faut de plus assurer la sécurité des échantillons (conservation dans un lieu approprié, sous clé, etc.). Les mesures de sécurité physique proposées dans le projet de recherche doivent être déployées et maintenues aussi longtemps que nécessaire.

Par ailleurs, tel que le recommande l'Énoncé de politique des trois Conseils, le chercheur prend aussi des engagements quant à la durée de 
conservation $^{73}$. Les échantillons sont alors conservés pour la durée nécessaire à la réalisation d'un projet de recherche ou pour une période fixe déterminée à l'avance. On peut penser qu'il s'agit d'une mesure visant en elle-même à assurer un certain contrôle et un certain suivi de l'utilisation des échantillons. L'échéance de ce délai force en effet à reconsidérer l'utilisation de l'échantillon pour empêcher qu'il ne tombe en désuétude ou encore que son usage dévie par rapport à ce qui avait originalement été convenu. Pour qu'un suivi puisse véritablement s'effectuer à ce sujet, il est indispensable de disposer d'un registre indiquant l'échéance des différentes banques au sein d'une institution donnée afin de ne pas passer sous silence ce moment important.

En outre, l'échantillon doit être conservé de façon à préserver l'identité de la personne dont il provient. Cette identité doit être tenue dans les plus strictes limites de la confidentialité par le chercheur et son équipe. Différentes méthodes peuvent alors être employées, allant de l'anonymisation à l'utilisation d'un code liant l'échantillon au dossier du participant. Quelles que soient les mesures mises en place, le CÉR doit être satisfait des mesures employées. Or, il est utile de vérifier si le plan élaboré dans le protocole de recherche est réellement mis en place et s'il s'avère aussi efficace qu'anticipé.

Finalement, la saine gestion d'une banque d'échantillons d'ADN ou de tissus a pour corollaire la gestion adéquate des demandes d'accès par des personnes autres que l'équipe de recherche. Les demandes d'accès devraient être documentées dans un registre et faire l'objet d'un suivi.

On constate donc qu'un suivi de la bonne conservation des échantillons est requis. Cependant, il n'est pas évident que le CÉR soit en mesure d'en assumer toutes les facettes. Le domaine de la qualité et des mesures de sécurité physique des échantillons n'est pas du ressort ni même de la compétence des CÉR. On ne pourrait imaginer que les membres de comité d'éthique s'assurent que les frigos sont barrés et à la bonne température! En fait, la responsabilité incombe plus directement à l'institution qui les héberge et à d'autres autorités plus compétentes en la matière. Cependant, en cas d'incident causant la perte ou la destruction d'échantillons, de tels événements devraient être rapportés au

73. Énoncé de politique, supra note 14 , règle 8.6. 
CÉR, un peu à la manière d'un effet sérieux indésirable. En effet, une telle situation peut indiquer que les mesures de conservations ne sont pas adéquates, ce qui ne serait pas éthique.

Par ailleurs, le sort réservé aux échantillons et tout événement qui suit l'échéance de la banque devraient, à tout le moins, être rapportés au CÉR sous la forme d'un rapport. Par exemple, le banquier devrait faire état de la destruction des échantillons d'ADN effectuée à la demande d'un participant. Enfin, en ce qui a trait au respect des autres engagements visant à préserver la confidentialité des participants, le CÉR doit au moins vérifier les rapports témoignant d'une mise en œuvre conforme aux mesures annoncées dans le protocole de recherche et dans la politique de mise en banque. Il serait aussi souhaitable que des vérifications sur le terrain puissent être effectuées pour vérifier cette mise en œuvre et juger, de visu, de son efficacité. Cependant, il faudrait déployer des ressources financières pour permettre de telles vérifications à propos de l'éthique d'un projet.

\section{c) Le respect des engagements pris à l'égard du déroulement de la recherche et de l'utilisation des échantillons}

Lorsqu'un participant prête son concours à un projet de recherche en génétique, il peut avoir accepté diverses options quant à l'utilisation qui en sera faite. Son échantillon peut ne servir qu'à un seul projet, à un programme de recherche, à une équipe de recherche internationale ou même à toutes fins jugées utiles pour la poursuite d'autres recherches acceptables par un CÉR.

Il est important de ne pas trahir l'entente initiale en dénaturant l'orientation du projet auquel le participant a consenti. Les travaux de recherche doivent demeurer dans la ligne de pensée à laquelle un participant s'est associé par son consentement ; le CÉR doit y veiller. Un rapport soumis au CÉR permet de connaître l'utilisation qui est faite des échantillons et d'endosser la poursuite de ces recherches par des 
«réapprobations» annuelles ${ }^{74}$. Ce rapport est particulièrement important dans le cas d'une banque à usages multiples.

À l'échéance du projet, plusieurs questions se posent. Qu'arrive-t-il avec les échantillons une fois le projet complété? Seront-ils utilisés à d'autres fins? Doit-on recontacter les participants? Le consentement indique peut-être qu'ils seront détruits, auquel cas quelqu'un doit veiller à ce que ce soit fait. Si nécessaire, on peut envisager de prolonger ou de changer l'orientation du projet de recherche. Ce genre de décision ne peut se prendre qu'avec la collaboration et l'assentiment du CÉR et un consentement approprié. Par ailleurs, si rien n'est fait à l'échéance, il y a là aussi une situation qui mérite d'être soulignée par le CÉR pour éviter que la banque ne tombe dans la désuétude. Les mutations et fusions de banques commandent également un suivi, voire une surveillance étroite.

\section{d) Le respect des engagements pris à l'égard des résultats de recherche}

Les résultats généraux de la recherche doivent faire l'objet d'une diffusion publique. D'ailleurs, la Déclaration d'Helsinki enjoint aux intervenants de publier les bons et moins bons résultats de la recherche ${ }^{75}$. Les CÉR devraient porter une attention au sujet des publications qui sont effectuées suite à un projet de recherche. Il en est de même des produits commerciaux issus d'un projet. Toutefois, il faut dire que ce suivi est particulièrement difficile à effectuer compte tenu que ces résultats peuvent n'être disponibles que plusieurs années après le prélèvement d'ADN chez un participant.

Le suivi le plus étroit doit plutôt porter sur la diffusion de résultats personnels. Tout comme pour les échantillons eux-mêmes, la confidentialité des résultats individuels de recherche doit être assurée. Les demandes d'accès par les tiers doivent être gérées avec vigilance. La loi impose à cet égard une

74. Pour être à même de fournir un tel rapport, le chercheur doit lui-même tenir un registre de l'utilisation qui est faite des échantillons.

75. Association médicale mondiale, Déclaration d'Helsinki, supra note 25, art. 27. 
obligation de confidentialité qui limite la divulgation de ces données ${ }^{76}$. Les circonstances d'une telle divulgation doivent être déterminées à l'avance dans la politique de mise en banque. Le CÉR peut assurer un suivi de la gestion appropriée de ces cas en regard de procédures préalablement approuvées.

Certains projets de recherche peuvent prévoir ou constater après coup qu'un retour de résultat personnel aux participants s'avère à propos. Il s'agit d'une situation délicate. Lorsqu'il n'était pas prévu de recontacter le participant, il est nécessaire de soumettre la procédure de communication au CÉR (présenter les modes et les canaux de communication avec le participant). Par exemple, une simple lettre n'est peut-être pas appropriée en regard de la gravité des informations soumises au participant. Par ailleurs, lorsque ce contact était prévu dans le formulaire de consentement, il doit s'effectuer de façon correcte et selon une procédure éthique ${ }^{77}$. Le CÉR doit s'assurer que le chercheur a mis en place une procédure appropriée. Dans son rapport annuel, le chercheur devrait indiquer si des participants ont été informés de tels résultats et si les mesures déployées se sont avérées adéquates.

\section{Conclusion}

La surveillance éthique continue s'inscrit véritablement dans le mandat des CÉR. Elle doit s'effectuer non seulement dans le cadre des projets de recherche dans le domaine de la pharmaceutique mais aussi dans les projets de recherche en génétique avec mise en banque d'ADN. Cela demande toutefois au CÉR de porter son attention au-delà de la sécurité physique des participants pour toucher la confidentialité et le respect des volontés exprimées. De nouvelles questions doivent être formulées et posées aux chercheurs et aux

76. La règle générale énoncée dans le Code civil du Québec est claire et s'applique autant à un dossier de recherche en génétique ou à tout autre dossier contenant des informations personnelles au sujet d'une autre personne : «art. 37 . Toute personne qui constitue un dossier sur une autre personne doit avoir un intérêt sérieux et légitime à le faire. Elle ne peut recueillir que les renseignements pertinents à l'objet déclaré du dossier et elle ne peut, sans le consentement de l'intéressé ou l'autorisation de la loi, les communiquer à des tiers ou les utiliser à des fins incompatibles avec celles de sa constitution; elle ne peut non plus, dans la constitution ou l'utilisation du dossier, porter autrement atteinte à la vie privée de l'intéressé ni à sa réputation.»

77. Par exemple, cette procédure doit comprendre un conseil génétique. 
promoteurs. Des ententes avec les banquiers et chercheurs impliqués sont aussi nécessaires pour établir les conditions d'utilisation et de mise en banque d'échantillons d'ADN. Dans un domaine de recherche en pleine effervescence, la surveillance éthique des projets de recherche en génétique doit soutenir et stimuler la qualité éthique des projets de recherche.

Les projets de recherche menant à la création d'une banque d'ADN qui sera conservée dans une autre institution ${ }^{78}$ que celle où siège le CÉR posent toutefois des défis particuliers pour lesquels ce dernier est souvent mal outillé. Visiblement, la capacité réelle des CÉR d'effectuer un suivi au sujet de ces banques a des limites. De plus, il est permis de douter de l'efficacité d'activités de suivi morcelées, mal coordonnées ou trop éloignées de ce qui se passe «sur le terrain». Enfin, l'absence d'une gestion centralisée des activités de suivi de l'éthique d'un tel projet de recherche crée des conditions propices à l'existence de zones grises où l'identification d'entorses à l'éthique est difficile.

Dans le cas, des banques créées, à long terme, à partir d'échantillons recueillis dans une multitude de centres, il y a peut-être lieu d'examiner des alternatives plus réalistes que l'unique recours aux CÉR locaux. La gouvernance de ces projets requiert peut-être la mise en place de modèles novateurs. Par exemple, la Commission d'accès à l'information pourrait jouer un rôle accru dans la surveillance des aspects liés à la confidentialité des banques d'ADN. On pourrait aussi imaginer la création d'une entité neutre dédiée à la surveillance des activités futures de la banque, pouvant veiller aux intérêts des participants à long terme et capable d'exiger la reddition de compte de la part des banquiers et de faire enquête, le cas échéant ${ }^{79}$. On pourrait aussi créer des structures législatives pour prêter main forte aux CÉR locaux en énonçant l'obligation juridique de faire rapport des activités liées à l'existence d'une banque d' $\mathrm{ADN}^{80}$. Quelle que soit la solution envisagée, il faudra veiller à conserver la perspective

78. Particulièrement si les échantillons seront échangés avec d'autres chercheurs.

79. Par exemple, on tente de créer, au sein de l'Université de Montréal, l'Institut de population et de génétique qui pourrait être appelé à jouer un rôle de cet ordre à l'égard de projets en génétique des populations, comme par exemple le projet Cartagène.

80. Tel que recommandé dans l'Avis de la Commission de l'éthique de la science et de la technologie (précité). 
éthique dans le suivi qui sera effectué ${ }^{81}$ et ne pas transformer le suivi en un simple exercice administratif.

En outre, le suivi requiert des ressources financières qui présentement ne sont pas au rendez-vous. Pourtant, on doit accorder autant d'importance à l'éthique de la recherche qu'à la rigueur scientifique qui fait la réputation de nos chercheurs. Le maintient des plus hauts standards en éthique de la recherche est nécessaire pour que les participants mettent en toute confiance leur patrimoine génétique au service de la science et du bien-être de la collectivité. Sachant que ce précieux lien de confiance existe généralement entre la population québécoise et la communauté scientifique, il y a tout lieu d'investir pour le préserver.

81. Un simple audit des données de recherche ne suffit pas à assurer le suivi éthique. 